\title{
Drug Reaction with Eosinophilia and Systemic Symptoms associada ao Alopurinol: um relato de caso
}

\author{
Allopurinol-associated with Drug Reaction with Eosinophilia and Systemic Symptoms: a \\ case report
}
Drug Reaction with Eosinophilia and Systemic Symptoms asociado a Alopurinol: reporte de un caso

Luelen da Silva Pinto ${ }^{1}$, Arthur Vinícius da Costa Paes Leme ${ }^{2}$, Brendha Camargo ${ }^{2 *}$, Bruna Moreira da Silva², Camila Teresa da Silva Santos², Wilson Cunha Junior².

\section{RESUMO}

Objetivo: Relatar um caso de Drug Reaction with Eosinophilia and Systemic Symptoms (DRESS) associada ao Alopurinol, uma vez que se trata de uma condição clínica rara e está associada a elevada morbimortalidade. Detalhamento do caso: Paciente mulher, 69 anos, internada em hospital terciário do interior de São Paulo, com febre há 10 dias e exantema difuso por todo corpo associado a prurido generalizado. Aos exames laboratoriais, havia eosinofilia e leucocitose. Fazia uso do medicamento Alopurinol há três meses. Iniciou-se o tratamento com corticoterapia, apresentando melhora parcial do exantema. No oitavo dia de internação, foi feita a hipótese de síndrome DRESS e aumentada a dose de prednisona, com melhora completa do quadro. A biópsia de pele demonstrou achado histológico discretos e inespecíficos. Paciente recebeu alta com encaminhamento para seguimento ambulatorial, com a finalidade do desmame da corticoterapia. Considerações finais: $O$ reconhecimento e tratamento precoces da doença são essenciais para melhorar o prognóstico dos pacientes doentes e consequentemente diminuir a morbimortalidade da doença.

Palavras-chave: Hipersensibilidade a drogas, Relatos de caso, Registros médicos.

\section{ABSTRACT}

Objective: To report a case of Drug Reaction with Eosinophilia and Systemic Symptoms (DRESS) associated with allopurinol, since it is a rare clinical condition and is associated with high morbidity and mortality. Details of the case: A 69-year-old female patient, admitted to a tertiary hospital in the interior of São Paulo, with fever for 10 days and a diffuse rash all over her body associated with generalized pruritus. Laboratory tests showed eosinophilia and leukocytosis. He had been using the drug Allopurinol for three months. Treatment with corticotherapy was started, showing partial improvement of the rash. On the eighth day of hospitalization, the hypothesis of DRESS syndrome was made and the dose of prednisone was increased, with complete improvement of the condition. Skin biopsy showed mild and nonspecific histological findings. The patient was discharged with an outpatient referral for the purpose of weaning from corticosteroid therapy. Final considerations: Early recognition and treatment of the disease are essential to improve the prognosis of sick patients and consequently reduce the morbidity and mortality of the disease.

Key words: Drug hypersensitivity, Case reports, Medical reports.

\section{RESUMEN}

Objetivo: Reportar un caso de Reacción a Fármaco con Eosinofilia y Síntomas Sistémicos (DRESS) asociado al alopurinol, ya que es una condición clínica rara y se asocia con alta morbimortalidad. Detalles del caso: Paciente femenina de 69 años, ingresada en un hospital terciario del interior de São Paulo, con fiebre de 10 días y un exantema difuso en todo el cuerpo asociado a prurito generalizado. Las pruebas de laboratorio mostraron eosinofilia y leucocitosis. Había estado usando el fármaco alopurinol durante tres meses. Se inició tratamiento con corticoterapia, mostrando mejoría parcial del exantema. Al octavo día de internación se planteó la hipótesis de síndrome DRESS y se aumentó la dosis de prednisona, con mejoría completa de la

${ }^{1}$ Fundação Santa Casa de Misericórdia de Franca, Franca - SP.

2 Universidade de Franca (UNIFRAN), Franca - SP. *E-mail: brecamargo1@gmail.com 
condición. La biopsia de piel mostró hallazgos histológicos leves e inespecíficos. El paciente fue dado de alta con una derivación ambulatoria con el fin de dejar de recibir tratamiento con corticosteroides. Consideraciones finales: El reconocimiento y tratamiento precoces de la enfermedad son fundamentales para mejorar el pronóstico de los pacientes enfermos y, en consecuencia, reducir la morbilidad y mortalidad de la enfermedad.

Palabras clave: Hipersensibilidad a las drogas, Informes de casos, Registros médicos.

\section{INTRODUÇÃO}

A síndrome de reação a medicamentos com eosinofilia e sintomas sistêmicos ou Drug Reaction with Eosinophilia and Systemic Symptoms (DRESS), conhecido como Síndrome da Hipersensibilidade Induzida por Droga é uma doença incomum que ocorre em cada 1000 a 10000 exposições a medicações (CRIADO PR, et al., 2012; GARZA JO, et al., 2015).

Caracteriza-se por início tardio, geralmente de três semanas a três meses após a introdução da droga, englobando como principais e primeiros sintomas a febre alta e a erupção cutânea maculopapular ou dermatite esfoliativa (CHAPMAN S, et al., 2020). Também são comuns o edema, a linfadenopatia, alterações hematológicas como eosinofilia (maior que 20000 por $\mathrm{mm}^{3}$ ), leucocitose (até 50000 por $\mathrm{mm}^{3}$ ) e atipia de linfócitos como na mononucleose-like, além de acometimento de multiorgânico (fígado, coração, rins, vasos, encéfalo, dentre outros) (CRIADO PR, et al., 2012).

As mortes giram em torno de $20 \%$ dos casos e as principais drogas relacionadas com o desenvolvimento dessa síndrome são anticonvulsivantes (fenitoína, fenobarbital e carbamazepina), dapsona, alopurinol, bloqueadores dos canais de cálcio, sulfonamidas, anti inflamatórios não esteroidais (AINEs), antirretrovirais, terbinafina e minociclina (GARZA JO, et al., 2015).

Tendo em vista a síndrome desencadeada pelo uso do alopurinol, a incidência é de apenas $2 \%$ ou um a cada 260 pacientes, ou seja, é uma reação de hipersensibilidade incomum, assim como no contexto geral da síndrome de DRESS (CRIADO PR, et al., 2012; MARKEL A, 2005).

A fisiopatologia exata dos mecanismos de desenvolvimento dessa doença ainda não é bem elucidada, mas acredita-se que ocorram mecanismos imunológicos que causam danos tecidual, principalmente reação de hipersensibilidade do tipo IV ao alopurinol e ao oxipurinol, metabólito cujo acúmulo parece ser significante em pessoas com insuficiência renal. Polimorfismos nos genes do sistema HLA (Human Leukocyte Antigens) podem explicar a predisposição genética de alguns indivíduos para desenvolverem síndrome de DRESS (CRIADO PR, et al., 2012; MARKEL A, 2005).

A hipótese mais aceita para explicar a interação entre o sistema imune e os fármacos é a teoria do hapteno/pro-hapteno. Segundo este modelo explicativo, o fármaco, ou o seu metabolito, liga-se de forma covalente a uma proteína endógena que, posteriormente, sofre uma série de processos intracelulares dos quais resultam uma série de peptídeos modificados quimicamente.

Estes peptídeos modificados, quando apresentados por uma célula apresentadora de antígenos, são reconhecidos como "estranhos" pelos receptores das células $T$, o que leva à ativação e proliferação dos linfócitos $\mathrm{T}$. Com isso, ocorre uma resposta imune que poderá também incluir uma resposta das células $\mathrm{B}$, com formação de anticorpos. (CRIADO PR, et al., 2012; WHITE K, et al., 2015). Outra possível alteração presente nesse contexto é a falha nos mecanismos de "proteção" contra a toxicidade dos metabólitos de reações oxidativas da droga (MOCKENHAUPT M e PAULMANN M, 2018).

O diagnóstico é feito através da documentação do uso do alopurinol e a presença de dois critérios maiores ou um critério maior associado a um critério menor (SASIDHARANPILLAI S, et al., 2019). Os critérios maiores são piora da função renal, lesão hepática aguda e erupção cutânea; já os critérios menores são leucocitose, eosinofilia e febre (MARKEL A, 2005). Os principais diagnósticos diferenciais que devem ser feitos são síndrome de Stevens-Johnson, necrólise epidermoide tóxica (NET) e reativação do herpes vírus 6 (CRIADO PR, et al., 2012; GARZA JO, et al., 2015; MARKEL A, 2005). 
Em relação ao tratamento, a primeira ação deve ser a retirada da medicação responsável pelos sintomas. O uso de corticoides diminui os sintomas, mas o acometimento hepático e da pele pode ainda persistir por semanas. O emprego de $\mathrm{N}$-acetilcisteína intravenosa tem sido documentado, com o intuito antioxidante e inibitório de reações imunes. Tal medicação é utilizada como expectorante e também como antídoto na intoxicação por paracetamol. A dessensibilização à droga é utilizada geralmente quando é necessário o controle da hiperuricemia sintomática (CRIADO PR, et al., 2012; GARZA JO, et al., 2015; MARKEL A, 2005; ENSINA LF, et al., 2009).

Com tudo isso, fica claro que o uso de medicamentos deve ser feito diante de indicações precisas, além de que deve ser ajustado conforme a função renal. Além disso, sabe-se que apesar da síndrome de DRESS ser uma entidade incomum, tem importância clínica e índice de mortalidade significativas (CRIADO PR, et al., 2012; GARZA JO, et al., 2015; MARKEL A, 2005).

\section{RELATO DE CASO}

Mulher, 69 anos de idade, branca, esteve internada em uma enfermaria de clínica médica em um hospital terciário do interior de São Paulo, com quadro de febre $\left(38-39^{\circ} \mathrm{C}\right)$ há 10 dias e exantema difuso, caracterizado por erupções cutâneas avermelhadas distribuídas por todo corpo, incluindo palma das mãos e planta do pés, associado a prurido generalizado. Apresentava também anasarca e queixa de tosse seca sem período do dia predominante, e sem fatores de melhora ou de piora. Paciente negava outras queixas.

Ao exame físico, estava em bom estado geral, normocorada e hidratada, ausência de linfonodos palpáveis, oroscopia sem alterações, aparelhos cardiovascular e respiratório e abdome, sem alterações, presença de edema $++++/ 4+$ em membros inferiores, superiores e em face. Além disso, apresentava exantema maculopapular difuso em face, tronco e membros (Figura 1).

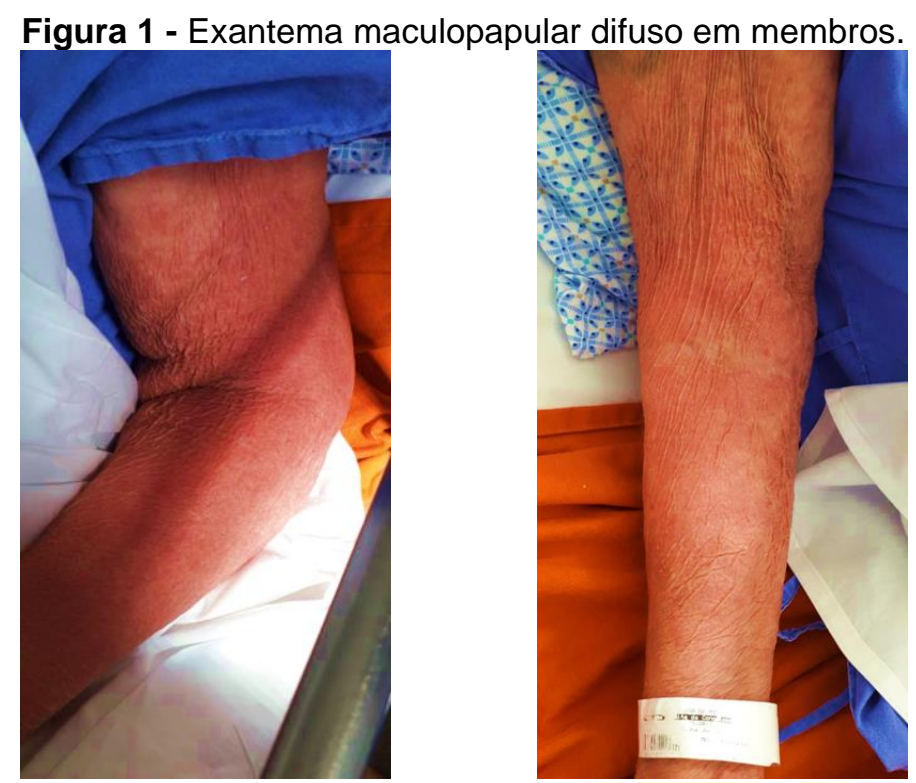

Fonte: PINTO LS, et al., 2020.

Paciente já havia passado por consulta anterior em pronto atendimento, sendo prescrito prednisona $20 \mathrm{mg} / \mathrm{dia}$ e loratadina $10 \mathrm{mg} / \mathrm{dia}$, sem melhora do quadro. De comorbidades apresentava asma controlada sem medicamento e deficiência cognitiva leve congênita. Fazia uso dos seguintes medicamentos: Alopurinol há três meses e Risperidona há três anos. Há cerca de um mês havia feito uso de amoxicilina para tratamento de pneumonia adquirida na comunidade.

Os exames da admissão apresentavam hemograma com plaquetopenia de $97.000 / \mathrm{mcL}$ (Valor de referência: $150.000-450.000 / \mathrm{mcL}$ ), leucocitose de $18.900 / \mathrm{mcL}$ (valor de referência: $4.500-10.000 / \mathrm{mcL}$ ) e eosinofilia de $5.481 / \mathrm{mcL}$ (valor de referência: $40-500 / \mathrm{mcL}$ ). Também havia elevação de transaminase glutâmico oxalacética (TGO) de $54 \mathrm{U} / \mathrm{L}$ (valor de referência: 5-40 U/L) e transaminase glutâmico pirúvica (TGP) de $116 \mathrm{U} / \mathrm{L}$ (valor de referência: 7-56 U/L). Demais exames sem alterações. 
No primeiro dia de internação foram suspensas as medicações de uso domiciliar e solicitada radiografia de tórax, sem alterações (Figura 2). Foram solicitadas também sorologias para dengue chikungunya e zika, porém não foram realizadas, já que a paciente estava fora dos critérios de epidemiologia necessários para realização dos exames.

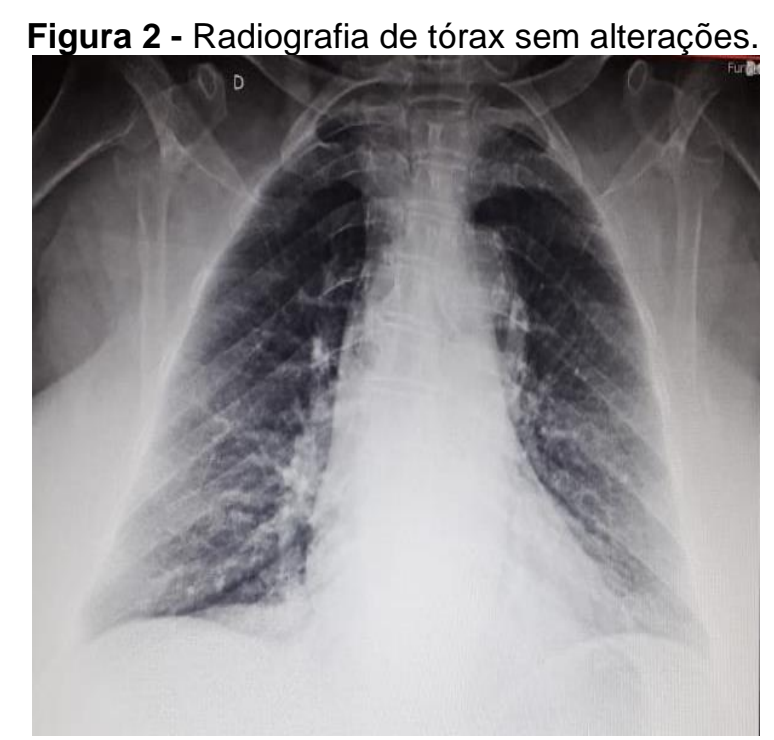

Fonte: PINTO LS, et al., 2020.

O exame de urina 1 demonstrou leucocitúria de 1.460.000/mL (valor de referência: 0 a $7.000 / \mathrm{mL}$ ), hematúria de $25.000 / \mathrm{mL}$ (valor de referência: 0 a $5.000 / \mathrm{mL}$ ) e bacteriúria aumentada. No hemograma, manteve leucocitose com desvio à esquerda, eosinofilia e plaquetas dentro dos valores de normalidade. Desse modo, foi iniciada antibioticoterapia com ceftriaxona, para tratamento de infecção do trato urinário.

Paciente evoluiu sem apresentar febre, porém teve alteração no padrão respiratório, apresentando queda da saturação de oxigênio e presença de sibilos na ausculta pulmonar, sendo necessário oxigenioterapia e iniciado tratamento com prednisona na dose de $40 \mathrm{mg} /$ dia. Após dois dias de corticoterapia, paciente iniciou melhora parcial do edema e do exantema, que evoluiu com descamação de pele tipo furfurácea (figura 3) e piora do prurido local.

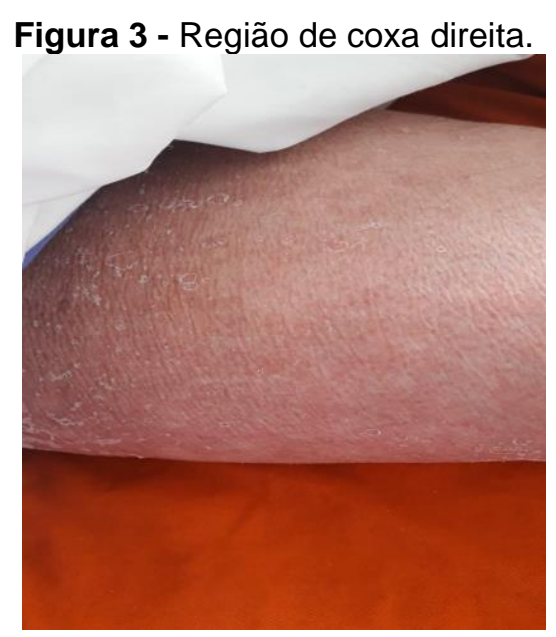

Fonte: PINTO LS, et al., 2020.

No oitavo dia de internação, foi feita a hipótese de síndrome DRESS e aumentada a dose de prednisona para $1 \mathrm{mg} / \mathrm{kg} / \mathrm{dia}(70 \mathrm{mg})$. A biópsia de pele constou o seguinte laudo: Fragmento de pele apresentando hiperceratose, epiderme atrófica com discretos focos de degeneração hidrópica basal. A derme mostra derrame pigmentar e leve infiltrado linfocitário superficial. Os achados histológicos são discretos e inespecíficos. 
Após isso, paciente apresentou melhora importante do exantema e do edema, porém ainda apresentava dependência de cateter de oxigênio para manter boa saturação. Foi aumentada a dose de prednisona para $1,5 \mathrm{mg} / \mathrm{kg} /$ dia e solicitado tomografia de tórax, a qual não demonstrou alterações.

Paciente evoluiu com desmame de oxigênio e melhora completa do exantema e do edema. Também apresentou melhora dos exames laboratoriais, com queda de eosinófilos. Recebeu alta com manutenção de prednisona e encaminhamento para ambulatório de clínica médica para desmame de corticoide.

\section{DISCUSSÃO}

A síndrome de DRESS, também conhecida como síndrome da hipersensibilidade induzida por fármacos, caracteriza-se por uma reação adversa rara, potencialmente fatal, podendo acometer tanto crianças quanto adultos, sendo estes mais afetados (MOCKENHAUPT M, 2017). Apresenta, na maioria dos casos, manifestações cutâneas e envolvimento de órgãos internos. Segundo o estudo prospectivo Registry of Severe Cutaneous Adverse Reaction (RegiSCAR) tem uma leve predominância no sexo feminino e em idades mais avançadas (CRIADO PR, et al., 2012).

O alopurinol é uma das principais medicações responsável pelo quadro (DESCAMPS V e RAGER-ROGEZ S, 2016), sendo a disfunção renal uma característica proeminente da síndrome de hipersensibilidade relacionada a esta medicação, afetando até $84 \%$ dos casos em comparação com outras formas de hipersensibilidade ao medicamento (que afeta menos de $30 \%$ ) e a mortalidade pode ser maior (LEE HY, et al., 2008)

O perfil da paciente corrobora o que se encontra na literatura acerca da epidemiologia da síndrome: é do sexo feminino e possui idade mais avançada (KURUVILLA M e KHAN DA, 2016). Apresentou sintomas após três meses do uso do alopurinol, identificando o início tardio do quadro. Em relação ao quadro clínico, apresentou alguns sintomas sugestivos da síndrome de DRESS, sendo eles a febre alta e exantema difuso, que configuram os primeiros sinais da doença (SÁNCHEZ-JAREÑO M, et al., 2018).

Além disso, surgiram alterações importantes no hemograma: leucocitose, eosinofilia, alteração da função renal e hepática. A eosinofilia é a anormalidade hematológica mais frequentemente encontrada ( $>50 \%$ dos casos) (SPRIET S, BANKS TA, 2015). Porém, outras anormalidades hematológicas observadas são trombocitopenia e anemia. Houve piora da função renal até iniciar a corticoterapia, o que indica fortemente uma possível degradação renal devido ao uso do alopurinol (AGUADO-VÁZQUEZ TM, et al., 2015).

O envolvimento hepático também é sugerido devido aos valores de transaminases duas vezes superior ao de normalidade, o que pode refletir uma lesão hepática aguda. Portanto, o comprometimento hepático e renal reforça o padrão sistêmico encontrado nesta condição clínica.

O diagnóstico de síndrome de DRESS associada ao alopurinol é feito através da documentação do uso desta medicação, ausência de exposição a outra droga que pode gerar quadro semelhante, a presença de pelo menos dois critérios maiores, ou a associação de um critério maior e um critério menor. Os critérios maiores incluem piora da função renal, lesão hepatocelular aguda e erupção cutânea, manifestada por necrólise epidérmica tóxica, eritema multiforme, erupção cutânea maculopapular difusa ou dermatite esfoliativa. Os critérios menores incluem febre, leucocitose e eosinofilia (MARKEL A, 2005). Como mencionado, a paciente fez uso da medicação, apresentou três dos critérios maiores, além de três menores; também não foi registrado o uso de outra medicação que poderia ocasionar os sintomas. Portanto, 0 diagnóstico de síndrome DRESS por alopurinol pode ser firmado.

Além disso, a análise histopatológica do tecido cutâneo e de outros órgãos podem ajudar na confirmação do diagnóstico. Apesar de não serem específicas da doença em questão, as alterações encontradas na biópsia de pele como o derrame pigmentar e presença de um infiltrado linfocitário são sugestivas de um quadro de farmacodermia (BUGNI VM, et al., 2008).

As lesões cutâneas são as apresentações mais prevalentes, estando presentes em 73 a $100 \%$ dos pacientes. Normalmente, a erupção cutânea envolve mais da metade da área da superfície corporal e pode até evoluir para eritrodermia. As lesões são geralmente pápulas e placas infiltrativas com alteração 
marcadamente purpúrica. Essas lesões cutâneas são frequentemente de apresentações polimórficas, que podem ser relatadas como lesões maculopapular, urticariforme, esfoliativa, liquenóide, pustular, bolhosa, tipo alvo ou tipo eczema (CHO Y-T, et al., 2017).

O edema facial, que pode ser encontrado em $76 \%$ dos pacientes, é uma característica marcante da doença. Mais tarde, a descamação se apresenta no estágio de resolução. O quadro de acometimento cutâneo da paciente do estudo configura um exemplo de caso que evoluiu com edema facial importante, com erupção tipo maculopapular pruriginosa e que posteriormente evoluiu para descamação tipo furfurácea ( $\mathrm{CHO}$ Y-T, et al., 2017; CHEN Y-C, et al., 2010).

O tratamento ainda não é padronizado. Porém, a retirada imediata dos medicamentos estimulantes é a ação mais importante a ser tomada no manejo de pacientes com síndrome de DRESS. Os sintomas cutâneos podem ser amenizados com a introdução de corticoterapia sistêmica, que geralmente traz resultados satisfatórios. A medicação recomendada é a prednisona, iniciada na dose de 40-60 mg/dia, sendo que tal dose deve ser reduzida gradualmente ao longo de 3 a 6 meses, após controle clínico e laboratorial. Essa medida tem como objetivo evitar recidiva dos sintomas e do conhecido efeito-rebote dos glicocorticoides (CHEN Y-C, et al., 2010; SHIOHARA T, et al., 2012).

A evolução clínica e laboratorial dos pacientes com síndrome de DRESS geralmente é favorável, com melhora significativa dos sintomas após a retirada do fármaco causal e introdução da prednisona no esquema terapêutico, como apresentou a paciente do presente estudo. Entretanto, alguns casos podem evoluir com complicações permanentes, que pode ser desde alterações pigmentares e cicatrizes cutâneas até alterações endócrinas, como o desenvolvimento de um transtorno da tireoide, já que este é órgão mais comumente afetado a longo prazo (FERREIRA MIP, et al., 2017).

Após a estabilidade do quadro da paciente, ela teve alta e foi encaminhada ao ambulatório de clínica médica, devido à necessidade de adequação da dose do glicocorticoide prescrito, de modo a progredir o seu desmame gradualmente, além do acompanhamento de possíveis complicações como as citadas acima.

Analisando o tratamento empregado em outro caso de síndrome de DRESS disponível na literatura, o paciente do estudo obteve melhora significativa tanto dos sintomas cutâneos quanto da função renal e hepática após o início da corticoterapia e suspensão da droga causadora dos sintomas. Além disso, foi encaminhado a um ambulatório de medicina interna para seguimento, corroborando com a conduta empregada a paciente do caso aqui relatado.

Pacientes e médicos devem ser educados sobre o reconhecimento precoce da hipersensibilidade ao medicamento e a importância da sua retirada imediata em tal evento (COSTA FSO, et al., 2019). Com essas recomendações, espera-se que a morbimortalidade grave associada à síndrome de hipersensibilidade ao alopurinol possa ser evitada (LEE HY, 2008).

Este relato de caso aborda um tema pouco discutido dentre as pesquisas médicas e foi realizado com intuito de ampliar o conhecimento e adicionar dados ao acervo de pesquisas sobre a síndrome de DRESS associada ao alopurinol, assim como também visa expandir o conteúdo sobre o assunto em vigor, já que se trata de uma doença pouco vista e a qual o diagnóstico ainda representa um desafio dentro do cenário médico. Dessa forma, conclui-se que seu reconhecimento e tratamento precoces são essenciais para melhorar o prognóstico dos pacientes doentes e consequentemente diminuir a morbimortalidade da doença.

\section{REFERÊNCIAS}

1. AGUADO-VÁZQUEZ TM, et al. Lamotrigine-associated drug reaction with eosinophilia and systemic symptoms (DRESS). Médica Sur, 2015; 22(3): 153-156.

2. BUGNI VM, et al. Síndrome de DRESS e Lupus eritematoso sistêmico juvenil em menina de dois anos de idade. Revista Brasileira de Reumatologia, 2008; 48(4).

3. CHAPAN S, et al. Apremilast-associated drug reaction with eosinophilia and systemic symptoms. Journal of the American Academy of Dermatology Case Reports, 2020; 6(4): 302-304.

4. CHEN Y-C, et al. Drug reaction with eosinophilia and systemic symptoms: a retrospective study of 60 cases. Archives of Dermatology, 2010; 146(12): 1373-1379. 
5. $\mathrm{CHO}$ Y-T, et al. Drug reaction with eosinophilia and systemic symptoms (DRESS): an interplay among drugs, viruses, and immune system. International Journal of Molecular Sciences, 2017; 18(6).

6. CRIADO PR, et al. Reação a drogas com eosinofilia e sintomas sistêmicos (DRESS) / Síndrome da hipersensibilidade induzida por drogas (DIHS): revisão dos conceitos atuais. Anais Brasileiros de Dermatologia, 2012; 87(3): 435-449.

7. WHITE K, et al. Evolving models of the immunopathogenesis of T cell-mediated drug allergy: The role of host, pathogens, and drug response. Journal of Allergy and Clinical Immunology. 2015;136(2):219-234.

8. COSTA FSO, et al. Segurança do paciente: Percepção de profissionais de saúde de uma fundação pública estadual de Belém-PA. Revista Eletrônica Acervo Saúde, 2019; 11(9): e350.

9. DESCAMPS V, RAGER-ROGEZ S. Síndrome DRESS. EMC - Dermatología, 2016; 50(3): 1-9.

10. ENSINA LF, et al. Reações de Hipersensibilidade a Medicamentos - Parte III. Revista Brasileira de Alergia e Imunopatologia, 2009; 32: 178-183.

11. FERREIRA MIP, et al. DRESS: relato de caso com estudo genético. Arquivos de Asma, Alergia e Imunologia, 2017; 1(4): 417-421.

12. GARZA JO, et al. Reacción por drogas com eosinofilia y síntomas sistémicos (síndrome de DRESS). Estudio retrospectivo de nueve casos. Revista Médica Chile, 2015; 143(5): 577-583.

13. KURUVILLA M, KHAN DA. Eosinophilic Drug Allergy. Clinical Reviews in Allergy and Immunology, 2016; 50(2): 228239.

14. LEE HY. Ariyasinghe JTN. Thirumoorthy T. Allopurinol hypersensitivity syndrome: a preventable severe cutaneous adverse reaction? Singapore Medical Journal, 2008; 49(5).

15. MARKEL A. Allopurinol-induced DRESS syndrome. Israel Medical Association Journal, 2005; 7: 656-660.

16. MOCKENHAUPT M. Epidemiology of cutaneous adverse drug reactions. Allergol Select, 2017; 1(1): 96-108.

17. MOCKENHAUPT M, PAULMANN M. Severe skin reactions due to new medications. Hautarzt, 2018; 69(4): $278-289$.

18. SÁNCHEZ-JAREÑO $\mathrm{M}$, et al. DRESS en paciente con toxoplasmosis y síndrome antisintetasa. IBJ Clinical Pharmacology, 2018; 1(1).

19. SASIDHARANPILLAI S, et al. Predictors of disease severity in drug reaction with eosinophilia and systemic symptoms. Indian Journal of Dermatology, Venereology and Leprology, 2019; 85(3): 266-275.

20. SHIOHARA T, et al. Drug-induced hypersensitivity syndrome: recent advances in the diagnosis, pathogenesis and management. Adverse Cutaneous Drug Eruptions, 2012; 97: 122-138.

21. SPRIET S, BANKS TA. Drug reaction with eosinophilia and systemic symptoms syndrome. Allergy \& Asthma Proceedings, 2015; 36(6): 501-505. 